\title{
Rotational dynamics of CO solvated in small He clusters: a quantum Monte Carlo study
}

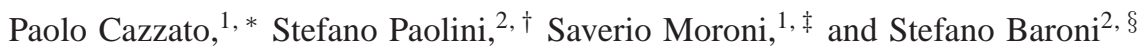 \\ ${ }^{1}$ SMC INFM - Istituto Nazionale per la Fisica della Materia and Dipartimento di Fisica, Università di Roma La Sapienza \\ Piazzale Aldo Moro 2, I-00185 Rome, Italy \\ ${ }^{2}$ SISSA - Scuola Internazionale Superiore di Studi Avanzati and INFM-DEMOCRITOS National Simulation Center, \\ via Beirut 2-4, I-34014 Trieste, Italy
}

(Dated: November 26, 2018)

\begin{abstract}
The rotational dynamics of CO single molecules solvated in small He clusters $\left(\mathrm{CO} @ \mathrm{He}_{N}\right)$ has been studied using Reptation Quantum Monte Carlo for cluster sizes up to $N=30$. Our results are in good agreement with the roto-vibrational features of the infrared spectrum recently determined for this system, and provide a deep insight into the relation between the structure of the cluster and its dynamics. Simulations for large $N$ also provide a prediction of the effective moment of inertia of $\mathrm{CO}$ in the He nano-droplet regime, which has not been measured so far.
\end{abstract}

PACS numbers: 36.40.-c, 34.20.+h, 67.40.Yv, 36.40.Mr, 02.70.Ss

\section{INTRODUCTION}

Thanks to recent progresses in Helium nano-droplet isolation (HENDI) spectroscopy, $\frac{1}{\stackrel{1}{1}}$ the infrared and microwave spectra of small molecules solvated in $\mathrm{He}_{N}$ clusters are now becoming accessible in the small- and intermediate-size regimes ${ }^{2,3,4.5}$ In the particular case of carbon monoxide, the roto-vibrational spectrum of the molecule solvated in small He clusters $\left(\mathrm{CO} @ \mathrm{He}_{N}\right)$ has been recently studied in the size range $N=2-20.5$ The infrared spectrum in the $2145 \mathrm{~cm}^{-1}$ region of the $\mathrm{C}-\mathrm{O}$ stretch consists of two $R(0)$ transitions which smoothly correlate with the $a$-type $(K=0 \leftarrow 0)$ and $b$-type $(K=1 \leftarrow 0) R(0)$ lines of the binary complex, CO@He $e_{1}$. The series of $b$-type transitions-which starts off about 7 times stronger for $N=1$-progressively looses intensity as $N$ increases, until it disappears around $N=7-8$. Around this size, just before it disappears, the $b$-type line seems to split in two. Analogously, around $N=15$ the $a$-type line also seems to split, and the assignment of experimental lines becomes uncertain for larger clusters. Elucidating the relation existing between the position, number, and intensity of the rotational lines and the size and structure of the cluster is the principal goal of the present paper.

Computer simulations of quantum many-body systems have also considerably progressed in recent years, allowing in some cases to determine the low-lying spectrum of excited states. The rotational dynamics of small molecules solvated in He clusters and nanodroplets is one of these favorable instances. The scarcity of low-lying excited states typical of superfluid systems makes it possible in this case to extract information on the location and intensity of the spectral lines from an analysis of the time series generated by quantum Monte Carlo random walks. .6 .8 The rotational spectrum of OCS@ $\mathrm{He}_{N}$ has been studied along these lines in Refs. [9 10]. Among the many different flavors of quantum Monte Carlo available in the literature, we adopt Reptation Quantum Monte Carlo ${ }^{6.7}$ which we believe presents distinctive advantages in the present case and which will be briefly introduced in Sec. III In Sec. IIII we present and discuss our results, whereas Sec. IV]contains our conclusions.

\section{THEORY, ALGORITHMS, AND TECHNICAL DETAILS}

Virtually all the ground-state quantum simulation methods are based on the prior knowledge of some approximate wave-function, $\Phi_{0}$, for the system under study. In the Variational Monte Carlo Method (VMC) one contents oneself of this knowledge and the simulation simply aims at calculating the complicated multi-dimensional integrals which are needed to estimate ground-state approximate expectation values, $\left\langle\Phi_{0}|\widehat{A}| \Phi_{0}\right\rangle$ (here and in the following quantummechanical operators are indicated with a hat, ${ }^{-}$). To this end, a random walk in configuration space is generated according to the Langevin equation:

$$
d \mathbf{x}=\epsilon \mathbf{f}_{0}(\mathbf{x})+d \boldsymbol{\xi},
$$

where $\mathbf{x} \equiv\left\{x_{\alpha}\right\}$ indicates the coordinates of the system, $\epsilon$ is the step of time discretization,

$$
\mathbf{f}_{0}(\mathbf{x})=2 \frac{\partial \log \left(\Phi_{0}(\mathbf{x})\right)}{\partial \mathbf{x}},
$$

and $d \boldsymbol{\xi}$ is a Gaussian random variable of variance $2 \epsilon$ : $\left\langle d \xi_{\alpha} d \xi_{\beta}\right\rangle=2 \epsilon \delta_{\alpha \beta}$. Approximate ground-state quantum expectation values are then estimated as time averages over the random walk, Eq. (1).

Within Reptation Quantum Monte Carlo (RQMC), exact ground-state expectation values and imaginary-time correlation functions are calculated as appropriate derivatives of the pseudo partition function, in the low temperature (large $T$ ) limit:

$$
\mathcal{Z}_{0}=\left\langle\Phi_{0}\left|e^{-T \widehat{H}}\right| \Phi_{0}\right\rangle
$$

where $\widehat{H}$ is the Hamiltonian of the system. By breaking the time $T$ into $P$ intervals of length $\epsilon=T / P$, Eq. (3) can be given a path-integral representation:

$$
\mathcal{Z}_{0} \approx \int \Phi_{0}\left(\mathbf{x}_{0}\right) \Pi_{i=0}^{P-1}\left\langle\mathbf{x}_{i}\left|e^{-\epsilon \widehat{H}}\right| \mathbf{x}_{i+1}\right\rangle \Phi_{0}\left(\mathbf{x}_{P}\right) d^{P+1} \mathbf{x} .
$$

For the relatively small systems considered here, it is sufficient to use the primitive approximation to the imaginary-time 
propagator:

$$
\left\langle\mathbf{x}\left|e^{-\epsilon \widehat{H}}\right| \mathbf{y}\right\rangle \propto \mathrm{e}^{-(\mathbf{x}-\mathbf{y})^{2} / 2 \epsilon-\epsilon[V(\mathbf{x})+V(\mathbf{y})] / 2}+\mathcal{O}\left(\epsilon^{3}\right),
$$

where $V(\mathbf{x})$ is the potential energy at point $\mathbf{x}$. The dynamical variables of the statistical-mechanical system whose partition function is given by Eq. (4) are segments of the VMC random walk generated from Eq. (1), $\mathbf{x}(\tau)$ of length $T$, which we call reptiles. As the random walk proceeds, the reptile is allowed to creep back and forth: new configurations of the reptile are accepted or rejected according to a Metropolis test made on the integrand of the path-integral representation of $\mathcal{Z}_{0}$, Eq. (4). It can be demonstrated ${ }^{6.7}$ that in the large- $T$ limit the sample of reptile configurations thus generated is such that the sample average of quantities like

$$
\mathcal{A}[\mathbf{x}(\tau)]=\frac{1}{T} \int_{0}^{T} A(\mathbf{x}(\tau)) d \tau
$$

converges without any systematic bias (but those due to the finite values of $\epsilon$ and $T$ ) to $\langle\widehat{A}\rangle=\left\langle\Psi_{0}|\widehat{A}| \Psi_{0}\right\rangle, \Psi_{0}$ being the exact ground-state wavefunction of the system. Even more interesting is the fact that sample averages of reptile time correlations,

$$
\mathcal{C}_{A}(\tau)=\frac{1}{T-\tau} \int_{0}^{T-\tau} A\left(\mathbf{x}\left(\tau^{\prime}\right)\right) A\left(\mathbf{x}\left(\tau^{\prime}+\tau\right)\right) d \tau^{\prime},
$$

provide equally unbiased estimates of the corresponding quantum correlation functions in imaginary time:

$$
\begin{aligned}
C_{\widehat{A}}(i t) & =\left\langle\Psi_{0}|\widehat{A}(i t) \widehat{A}(0)| \Psi_{0}\right\rangle \\
& \equiv\left\langle\Psi_{0}\left|\mathrm{e}^{\widehat{H} t} \widehat{A} \mathrm{e}^{-\widehat{H} t} \widehat{A}\right| \Psi_{0}\right\rangle,
\end{aligned}
$$

$\mathcal{C}_{A}(\tau) \approx C_{\widehat{A}}(i \tau)$. The absorption spectrum of a molecule solvated in a non polar environment is given by the Fourier transform of the autocorrelation function of its electric dipole, d:

$$
\begin{aligned}
I(\omega) & \propto 2 \pi \sum_{n}\left|\left\langle\Psi_{0}|\widehat{\mathbf{d}}| \Psi_{n}\right\rangle\right|^{2} \delta\left(E_{n}-E_{0}-\omega\right) \\
& \left.=\int_{-\infty}^{\infty} \mathrm{e}^{i \omega t} \widehat{\mathbf{d}}(t) \cdot \widehat{\mathbf{d}}(0)\right\rangle d t,
\end{aligned}
$$

where $\Psi_{0}$ and $\Psi_{n}$ are ground- and excited-state wavefunctions of the system respectively, and $E_{0}$ and $E_{n}$ the corresponding energies. The dipole of a linear molecule—such as $\mathrm{CO}-$ is oriented along its axis, so that the optical activity is essentially determined by the autocorrelation function of the molecular orientation versor: $c(t) \equiv C_{\widehat{\mathbf{n}}}(t)=$ $\left\langle\Psi_{0}\left|\mathrm{e}^{i \widehat{H} t} \widehat{\mathbf{n}} \mathrm{e}^{-i \widehat{H} t} \widehat{\mathbf{n}}\right| \Psi_{0}\right\rangle$. We have seen that RQMC gives easy access to the analytic continuation to imaginary time of correlation functions of this kind. From now on, when referring to time correlation functions, we will mean reptile time correlations, i.e. quantum correlation functions in imaginary time. Continuation to imaginary time transforms the oscillatory behavior of the real-time correlation function - which is responsible for the $\delta$-like peaks in its Fourier transform-into a sum of decaying exponentials whose decay constants are the excitation energies, $E_{n}-E_{0}$, and whose spectral weights are proportional to the absorption oscillator strengths, $\left|\left\langle\Psi_{0}|\mathbf{d}| \Psi_{n}\right\rangle\right|^{2}$. Dipole selection rules imply that only states with $J=1$ can be optically excited from the ground state which has $J=0$. Information on excited states with different angular momenta, $J$, can be easily obtained from the multipole correlation functions, $c_{J}(\tau)$, defined as the reptile time correlations of the Legendre polynomials:

$$
\begin{aligned}
c_{J}(\tau) & =\left\langle P_{J}(\mathbf{n}(\tau) \cdot \mathbf{n}(0))\right\rangle \\
& \equiv\left\langle\frac{4 \pi}{2 J+1} \sum_{M=-J}^{J} Y_{J M}^{*}(\mathbf{n}(\tau)) Y_{J M}(\mathbf{n}(0))\right\rangle
\end{aligned}
$$

Both the $\mathrm{He}-\mathrm{He}$ and the He-CO interactions used here

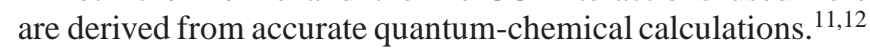
The CO molecule is allowed to perform translational and rotational motions, but it is assumed to be rigid. The trial wavefunction is chosen to be of the Jastrow form:

$$
\Phi_{0}=\exp \left[-\sum_{i=1}^{N} \mathcal{U}_{1}\left(r_{i}, \theta_{i}\right)-\sum_{i<j}^{N} \mathcal{U}_{2}\left(r_{i j}\right)\right],
$$

where $\mathbf{r}_{i}$ is the position of the i-th atom with respect to the center of mass of the molecule, $r_{i}=\left|\mathbf{r}_{i}\right|, \theta_{i}$ is the angle between the molecular axis and $\mathbf{r}_{i}$, and $r_{i j}$ is the distance between the $\mathrm{i}$-th and the $\mathrm{j}$-th helium atoms. $\mathcal{U}_{1}$ is expressed as a sum of five products of radial functions times Legendre polynomials. All radial functions (including $\mathcal{U}_{2}$ ) are optimized independently for each cluster size with respect to a total of 27 variational parameters. The propagation time is set to $T=1 \mathrm{~K}^{-1}$, with a time step of $\epsilon=10^{-3} \mathrm{~K}^{-1}$. The effects of the length of the time step and of the projection time have been estimated by test simulations performed by halving the former or doubling the latter. These effects were barely detectable on the total energy, and very small on the excitation energies discussed below (we estimate that more converged simulations would actually improve the already excellent agreement with experimentally observed spectra).

The estimate of excitation energies and spectral weights from imaginary-time correlations amounts to performing an inverse Laplace transform, a notoriously ill-conditioned operation which is severely hindered by statistical noise ${ }^{13}$ For each value of $J$, we extract the value of the two lowestlying excitation energies, $\epsilon_{a, b}^{J}$-i.e. the two smallest decay constants in $c_{J}(\tau)$-as well as the corresponding spectral weights, $A_{a, b}^{J}$, from a fit of $c_{J}(\tau)$ to a linear combination of three decaying exponentials. This fitting procedure does not solve in general the problem of obtaining the spectrum from a noisy imaginary-time correlation function. However, if we know in advance that very few strong peaks, well separated in energy, nearly exhaust the entire spectral weight, their position and strength can be reliably estimated from this multiexponential fit. In the present study these favorable conditions are usually met, although the limitations of the procedure will show in some cases, as discussed below. 


\section{RESULTS AND DISCUSSION}

RQMC simulations have been performed for $\mathrm{CO} @ \mathrm{He}_{N}$ clusters in the size range $N=1-30$. In Fig. 1 we report the values of the He atomic binding energy, $\Delta E_{N}=E_{N-1}-E_{N}$, as a function of the cluster size. $\Delta E_{N}$ first increases up to $N=4-5$, and it stays roughly constant in the range $N=5-8$; from this size on $\Delta E_{N}$ starts decreasing, first slowly, then, from $N=10-11$, rapidly down to a minimum at $N=19$. For $N>19 \Delta E_{N}$ increases again and slowly tends to the nanodroplet regime (where it coincides with the bulk chemical potential, $\mu=7.4 \mathrm{~K}^{7}$ ) which is however attained for much larger cluster sizes than explored here. ${ }^{14}$ This behavior can be understood by comparing the shape of the $\mathrm{CO}-\mathrm{He}$ potential energy function, $v(\mathbf{r})$, with the incremental atomic density distributions, $\Delta \rho_{N}(\mathbf{r})=\rho_{N}(\mathbf{r})-\rho_{N-1}(\mathbf{r})$, where $\rho_{N}$ is the expectation value of the He density operator:

$$
\widehat{\rho}(\mathbf{r})=\sum_{i=1}^{N} \delta\left(\mathbf{r}-\mathbf{r}_{i}\right)
$$

(see Fig. 2). For very small $N$ the atomic binding energy is dominated by the $\mathrm{He}-\mathrm{CO}$ attraction which is strongest in a well located atop the oxygen atom. As He atoms fill this well, $\Delta E_{N}$ first slightly increases, as a consequence of the attractive $\mathrm{He}-\mathrm{He}$ interaction, then, for larger $N$, the increased $\mathrm{He}-\mathrm{He}$ interaction is counter-balanced by the spill-out of $\mathrm{He}$ atoms off the main attractive well, until for $N \approx 9$ the reduction of the He-CO interaction overcomes the increased attraction and the binding energy starts decreasing steeply. For $N$ in the range 10-14 He density accumulates towards the $\mathrm{C}$ pole, while, around $N=15$, the first solvation shell is completed and the differential atomic density, $\Delta \rho_{N}$, is considerably more diffuse starting from $N=16 . \Delta E_{N}$ reaches a minimum at $N=19$. For larger sizes, the trend in the atomic binding energy is dominated by the increase of the He-He attraction related to the increase of the cluster size, until it will converge to the bulk chemical potential.

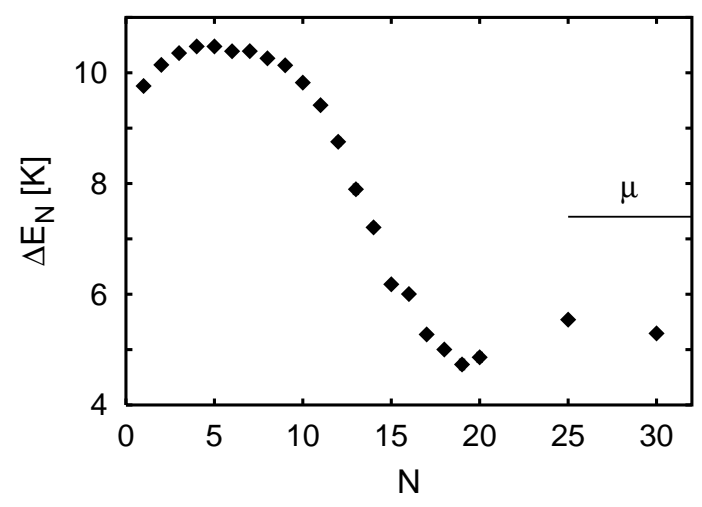

FIG. 1: Atomic binding energy, $\Delta E_{N}=E_{N}-E_{N-1}$, as a function of the cluster size in $\mathrm{CO} @ \mathrm{He}_{N}$. The horizontal line on the right of the figure indicates the chemical potential in bulk ${ }^{4} \mathrm{He}, \mu=7.4 \mathrm{~K}$ [7].

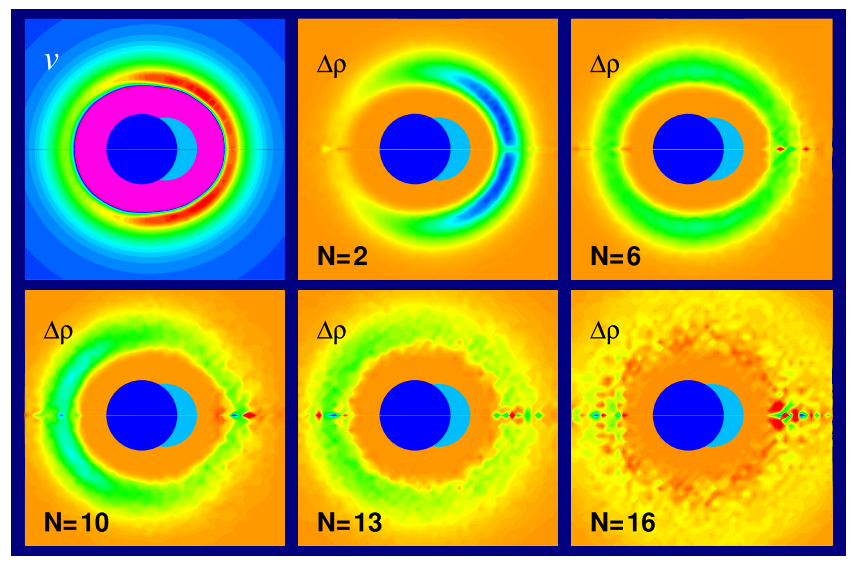

FIG. 2: (color) Upper left panel: He-CO interaction potential. C (blue) and $\mathrm{O}$ (cyan) atoms are pictured by two circles whose radius is the corresponding Van de Waals radius. The other panels picture the differential He density, $\Delta \rho_{N}=\rho_{N}-\rho_{N-1}$ for various sizes of the CO@ $\mathrm{He}_{N}$ cluster. Color convention is rainbow: red to purple in order of increasing magnitude.

In Fig. 3 we report the positions and spectral weights of the rotational lines, as functions of the cluster size, $N$. In the size range $N=1-9$, analysis of the dipole time correlations clearly reveals the presence of two peaks, with the weight of the higher-energy ( $b$-type) rapidly decreasing by almost a factor 2. Note that the sum of the spectral weights of these two lines nicely sums to one, indicating that they exhaust all the oscillator strength available for optical transitions originating from the ground state. For $N$ between 10 and 12 (shaded area in Fig. 3] the situation is less clear. As the weight of the $b$-type line drops to zero, the statistical noise on its position grows enormously. Furthermore the multi-exponential fit introduces some ambiguity, as the results are somewhat sensitive to the number of terms in the sum. However the important information that one line disappears between $N=10$ and 12 is clear. For larger $N$ only one relevant line remains, and the robustness of the fitting procedure is recovered, with the minor exception of the sizes around $N=16$, where the minimum of the $\chi^{2}$ appears to be less sharp, possibly correlating with the splitting of the line observed in the infrared spectra for $N=15$ (see below).

In the upper panel of Fig. 4 we compare the rotational structure of the observed infrared (vibrational) spectrum ${ }^{5}$ with the rotational excitation energies calculated in this work. Experimental data are referred to the center, $\nu_{0}$, of the vibrational band for $N=0$ (CO monomer). In order to better compare our predictions with experiments, we have corrected the former with an estimate of the vibrational shift, $\Delta \nu_{0}$, i.e. the displacement of the vibrational band origin as a function of the number of He atoms. The vibrational shift can be calculated as the difference in the total energy of the cluster obtained with two slightly different potentials,, $12 v_{00}$ and $v_{11}$, representing the interaction of a $\mathrm{He}$ atom with the $\mathrm{CO}$ molecule in its vibrational ground state and first excited states, respectively. Our estimate of the vibrational shift as a function of the cluster size 

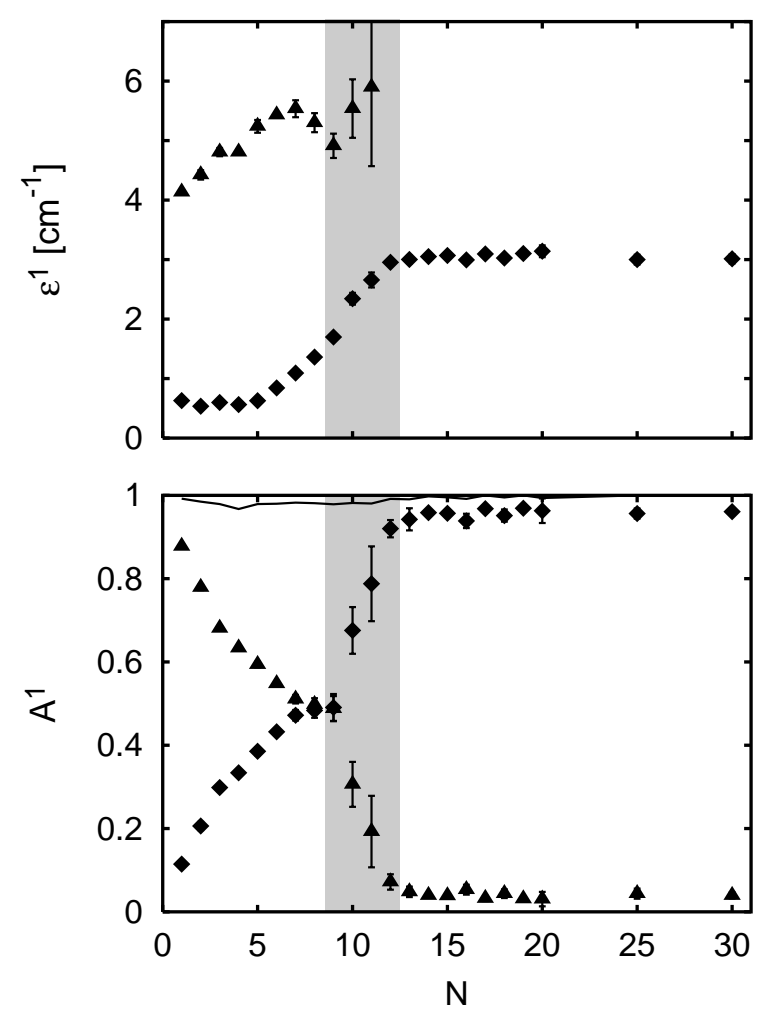

FIG. 3: Upper panel: position of the rotational lines of $\mathrm{CO} @ \mathrm{He}_{N}$ as obtained from RQMC simulations as a function of the cluster size, $N$. $a$-type lines are indicated with triangles, $b$-type lines with diamonds. Lower panel: spectral weights of the lines reported in the lower panel; the continuous line near the upper border of the figure corresponds to the sum of the spectral weights.

is reported in the lower panel of Fig. 14 Since the evaluation of a small difference between two large energies is computationally demanding for large clusters, $\Delta \nu_{0}$ has been evaluated perturbatively with respect to the difference $v_{00}-v_{11} \stackrel{14}{\underline{14}} \mathrm{We}$ have used the vibrational shift calculated in Ref. 14 after verifying on small clusters that the perturbative treatment is reliable. The agreement between our results and experiments is remarkable. Some of the features of the observed spectrum, however, call for a deeper understanding and theoretical investigation. Two questions, in particular, naturally arise. Why two peaks are observed in the small-size regime, and what determines the disappearance of one of them at $N=8$ ? What determines the split of the higher-frequency ( $b$-type) line at $N=7$ and of the lower-frequency ( $a$-type) one at $N=15$ ?

The existence of two lines for small $N$ is likely due to a larger asymmetry of the cluster in this regime. If the $\mathrm{CO} @ \mathrm{He}_{N}$ complex is described as a rigid rotor, in fact, one would have one rotational line originating from a $J=0$ ground state if the complex has cylindrical symmetry, while this line would double if some of the atomic density accumulates in a longitudinal protrusion. The inertia of the complex would in this case be larger for a rotation about an axis perpendicular to a plane containing the protrusion (end-over-end rotation) than about an axis lying on such a plane. Given that the $\mathrm{He}$ density in the ground state of $\mathrm{CO} @ \mathrm{He}_{N}$ is cylindrically
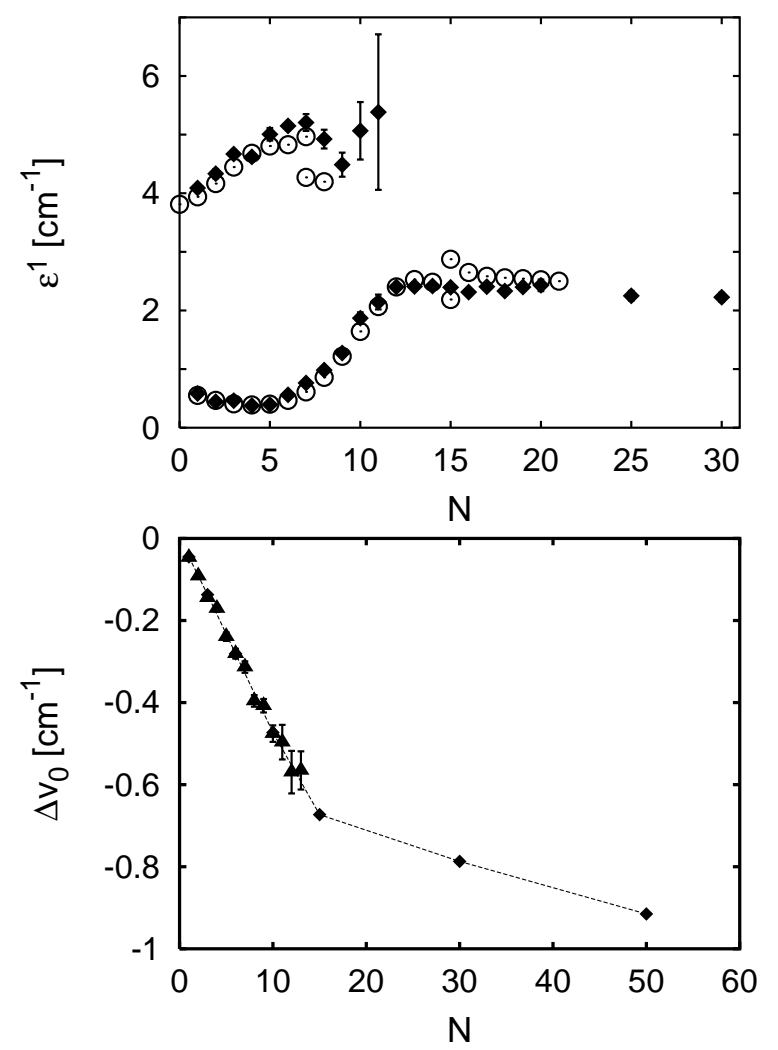

FIG. 4: Upper panel: positions of the infrared lines of CO@ $\mathrm{He}_{N}$ as observed experimentally (Ref. 5, empty circles) and as estimated from the present simulations and corrected by the estimated vibrational shift (solid diamonds, see text). Lower panel: vibrational shift of the lines, as estimated in the present work (triangles) and in Ref. 14 (diamonds and dashed line).

symmetric, any departure from this symmetry can only show up in higher correlation functions. The situation is conceptually similar to that of a fluid whose density is homogeneous and whose structure at the atomic scale is reflected in the pair correlation function. Analogously, we define an atomic angular correlation function, $C(\phi)$, as the probability of finding two He atoms which form a dihedral angle $\phi$ with respect to the molecular axis:

$$
C(\phi)=\left\langle\frac{1}{N(N-1)} \sum_{i \neq j} \delta\left(\phi_{i}-\phi_{j}-\phi\right)\right\rangle .
$$

In the upper panel of Fig. 5 we show $C(\phi)$, for different cluster sizes. The depletion of $C$ for $\phi$ larger than $\pi / 2$, clearly visible for $N=3$ (green circles), indicates a tendency of the $\mathrm{He}$ atoms to cluster on a same side of the molecular axis. For larger clusters, however, this effect weakens to the extent that it becomes difficult to disentangle from the structural information related to the $\mathrm{He}-\mathrm{He}$ interaction (the dimple at small $\phi$ and the subsequent maximum around $\phi=0.3-0.4 \pi)$. A more sensitive measure of the propensity of $\mathrm{He}$ atoms to cluster on a side of the molecule is given by the integral of $C(\phi)$ 

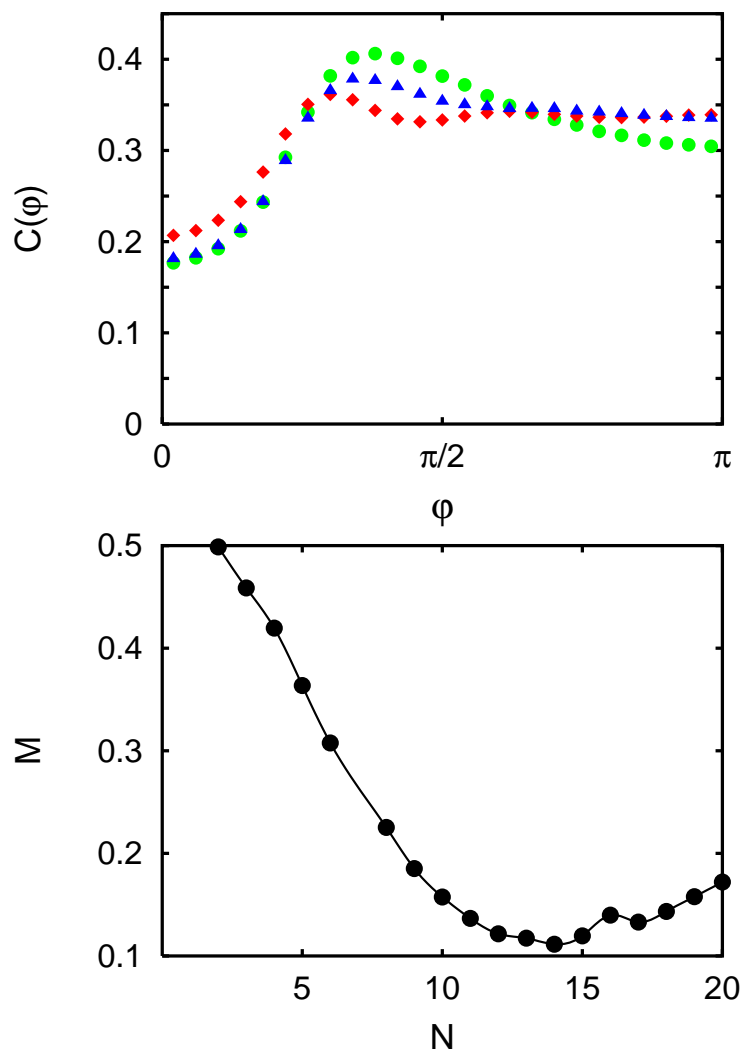

FIG. 5: (color) Upper panel: probability density of finding two $\mathrm{He}$ atoms which form a dihedral angle $\phi$ with respect to the molecular axis; the probability is normalized to 1 . Results pertain to clusters with $N=3$ (green circles), $N=6$ (blue triangles) and $N=13$ (red diamonds). Lower panel: the integrated probability density, defined in Eq. 14, as a function of the cluster size.

from 0 to $\frac{\pi}{2}$,

$$
M=\int_{0}^{\frac{\pi}{2}} C(\phi) d \phi-\frac{1}{2} .
$$

In the lower panel of Fig. 5 we display $M$ as a function of the cluster size, $N$ : one sees that $M$ decreases with $N$ and reaches a minimum at $N=14$. This is the size at which the first solvation shell is completed, and the cluster asymmetry increases again when the second shell starts to build. The rotational spectrum of the solvated molecule, however, is insensitive to this asymmetry for clusters of this and larger sizes because the motion of He atoms in the second and outer solvation shells is decoupled from that in the first and from molecular rotation. The existence of a longitudinal asymmetry is a necessary condition for the doubling of the rotational line. Whether or not this condition is also sufficient depends on the dynamics: if quantum fluctuations make the motion of the protrusion around the molecular axis fast with respect to the molecular rotation, then the asymmetry is effectively washed out. The existence of two lines in the rotational spectrum of the molecule implies therefore that an asymmetry in the classical distribution of $\mathrm{He}$ atoms around the molecular axis exists; that the molecular inertia is sensitive to this asym-

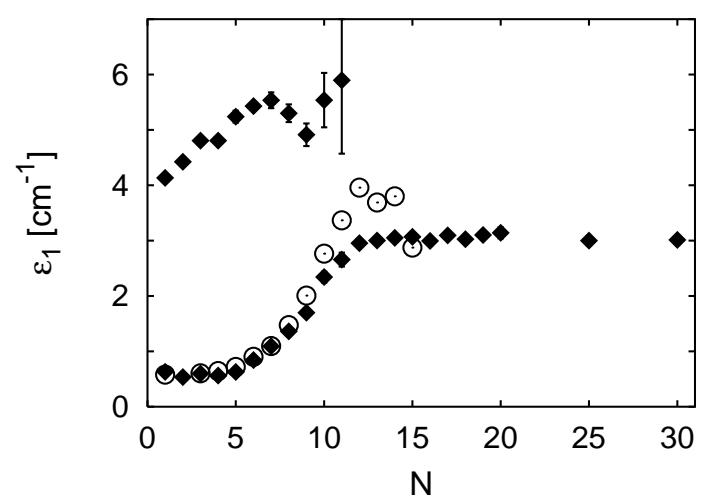

FIG. 6: Diamonds: CO rotational frequencies in $\mathrm{CO} @ \mathrm{He}_{N}$ as functions of the cluster size, $N$ (same as in Fig. 3. Dots: frequency of the lowest mode appearing in the spectral analysis of the angular $\mathrm{He}-\mathrm{He}$ correlation function (see Eq. 15).

metry (the protrusion can be 'dragged' along the molecular rotation); and that the motion of this protrusion around the molecular axis is not adiabatically decoupled from the molecular rotation.

In order to better characterize the motion of $\mathrm{He}$ atoms around the molecule and the coupling of this motion to molecular rotation, we examine the imaginary-time correlations of the versor, $\mathbf{u}$, of the He center of mass, $\mathbf{r}_{C M}$, relative to the molecular center of mass:

$$
\mathcal{C}_{\mathbf{u}}(\tau)=\langle\mathbf{u}(\tau) \cdot \mathbf{u}(0)\rangle
$$

For the binary complex, $\mathrm{He}-\mathrm{CO}, \mathbf{r}_{C M}$ coincides with the position of the helium atom, and we expect its angular dynamics to be strongly correlated to the molecular rotation, at least in the end-over-end mode. In Fig. 6 we report the frequency of the slowest mode appearing in the spectral analysis of $\mathcal{C}_{\mathbf{u}}(\tau)$, $\epsilon_{\mathbf{u}}$, as a function of $N$, and compare it with the corresponding frequencies of the molecular rotation. We see that for cluster sizes up to $N=9-10, \epsilon_{\mathbf{u}}$ is degenerate with the $a$-type frequency in the molecular rotational spectrum, with a spectral weight which passes from $A_{\mathbf{u}} \approx 1$ for $N=1$ to $A_{\mathbf{u}} \approx 0.7$ for $N=10$. These findings are a manifestation of the fact that $\mathrm{He}$ atoms are dragged along the slowest, end-over-end, rotation of the solvated molecule, and that the effect of this dragging decreases when more He states with $J=1$ become available and subtract spectral weight to the slowest mode. For $N>10$, $\epsilon_{\mathbf{u}}$ further increases and departs from $\epsilon_{a}$, indicating an effective decoupling of the two kinds of motion. In this regime, the effective rotational constant $B$ of the solvated molecule is almost independent of the cluster size. Free molecular rotation with an increased moment of inertia with respect to the gas phase is the typical signature of superfluid behavior in $\mathrm{He}$ nanodroplets. Extrapolating the result obtained for $N$ up to 30 to the nanodroplet limit, we predict a renormalization factor of the $B$ value of 0.78 . The lowest atomic mode, $\epsilon_{\mathbf{u}}$, slows down again for $N=15$. This is due, however to the slow $\mathrm{He}$ motion in the second solvation shell which hardly affects the rotation of the solvated molecule. Although the resolution that 
can be achieved with our simulations is not sufficient to detect the doubling of the $a$ and $b$ lines which is experimentally observed for $N=15$ and $N=7$ respectively, it is interesting to notice that the former occurs in correspondence with the crossing between $\epsilon_{\mathbf{u}}$ and $\epsilon_{a}$, possibly due to the resonant interaction between the two modes. It is tempting to assume that a similar mechanism may be responsible for the doubling of the $b$ line at $N=7$, involving however higher-energy $\mathrm{He}$ states. A deeper study of the He dynamics would clarify this point.

\section{CONCLUSIONS}

Computer simulations of quantum many-body systems have reached such a degree of sophistication and reliability that in some cases they can be used to provide information, complementary to that which can be obtained in the laboratory, on the dynamical processes probed spectroscopically.

In the case of small polar molecules solvated in He clusters, for instance, the calculation of the time autocorrelation of the molecular dipole (which is the quantity directly coupled to the experimental probe) allows to reproduce rather accurately the roto-vibrational excitation energies which are now becoming experimentally accessible for small clusters $(N=1-20)$. Even more importantly, computer simulations give direct access to quantitities and features (such as, e.g., static and dynamic properties of the He matrix) which are not accessible to the experiment, and whose knowledge provides the basis for understanding the relation between structure and dynamics in these confined boson systems.

In the specific case of $\mathrm{CO} @ \mathrm{He}_{N}$, which is the subject of the present study and of a recent infrared spectroscopy experiment,$\frac{5}{\underline{y}}$ the presence of two spectral lines- $a$-type and $b$-type, evolving respectively from the end-over-end and from the free-molecule rotations of the binary complex-is related to the propensity of the $\mathrm{He}$ atoms to cluster on a same side of the molecular axis, which we measure by an angular pair distribution function: as more $\mathrm{He}$ atoms progressively fill the first solvation shell, their clustering propensity weakens; the $\mathrm{CO}$ impurity gets more isotropically coated, looses a preferred axis for the free-molecule mode, and the $b$-type line disappears.

The time autocorrelation of the versor of the He center of mass provides dynamical information on the $\mathrm{He}$ atoms in excited states with $J=1$. We find a substantial spectral weight on a He mode whose energy, $\epsilon_{\mathbf{u}}$, is degenerate with the $a$-type line for $N$ up to about ten. This indicates that some of the $\mathrm{He}$ density is dragged along by the molecular rotation-in other words, part of the angular momentum in the cluster mode involving molecular rotation is carried by the He atoms. We also find that for larger clusters the molecular rotation effectively decouples from this He mode, and its energy $\epsilon_{a}$ becomes essentially independent of the number of He atoms. Based on the nearly constant value of $\epsilon_{a}$ in the range of $N$ between 15 and 30, well beyond completion of the first solvation shell, we predict the effective rotational constant in the nanodroplet limit to be smaller by a factor 0.78 than its gas phase value.

\section{Acknowledgments}

We would like to thank A.R.W. McKellar for providing us with a preprint of Ref. [5] prior to publication. We are grateful to G. Scoles for bringing that work to our attention, for his continuous interest in our work, and for many useful discussions. Last but not least, we wish to thank S. Fantoni for his encouragement and for a critical reading of our manuscript. This work has been partial supported by the Italian MIUR through PRIN.
* Electronic address: cazzato@democritos.it

$\dagger$ Electronic address: paolini@ sissa.it

¥ Electronic address: moroni@ caspur.it

$\S$ Electronic address: baroni@ sissa.it

${ }^{1}$ C. Callegari, K.K. Lehmann, R. Schmied and G. Scoles, J. Chem. Phys. 115, 10090 (2001).

2 J. Tang, Y. Xu, A.R.W. McKellar, and W. Jäger, Science 297, 1945 (2002).

3 Y.J. Xu and W. Jäger, J. Chem. Phys. 119, 5457 (2003).

4 J. Tang, A.R.W. McKellar, J. Chem. Phys. 119, 5467 (2003).

5 J. Tang, A.R.W. McKellar, J. Chem. Phys. 119, 754 (2003).

${ }^{6}$ S. Baroni and S. Moroni, in Quantum Monte Carlo Methods in Physics and Chemistry, edited by P. Nightingale and C.J. Umrigar. NATO ASI Series, Series C, Mathematical and Physical Sciences, Vol. 525, (Kluwer Academic Publishers, Boston, 1999), p. 313, also available at cond-mat/9808213

7 S. Baroni and S. Moroni, Phys. Rev. Lett. 82, 4745 (1999).

8 D. Blume, M. Lewerenz, P. Niyaz, and K. B. Whaley, Phys. Rev. E 55, 3664 (1997).

9 F. Paesani, A. Viel, F.A. Gianturco, and K.B. Whaley, Phys. Rev. Lett. 90, 73401 (2003).

10 S. Moroni, A. Sarsa, S. Fantoni, K.E. Schmidt, and S. Baroni, Phys. Rev. Lett. 90, 143401 (2003).

11 T. Korona, H. L. Williams, R. Bukowski, B. Jeziorski and K. Szalewicz, J. Chem. Phys. 106, 5109 (1997).

12 T. G. A. Heijmen, R. Moszynski, P. E. S. Wormer, and A. van der Avoird, J. Chem. Phys. 107, 9921 (1997).

13 J. E. Gubernatis and M. Jarrell, Phys. Rep. 269, 135 (1996).

${ }^{14}$ F. Paesani and F. A. Gianturco, J. Chem. Phys. 116, 10170 (2002). 